PAPER

\section{A note on the ubiquity of the traceless spin connection in dual massive spin- 2 theories}

To cite this article: D Dalmazi 2016 Class. Quantum Grav. 33075013

View the article online for updates and enhancements.

\section{Related content}

Hamiltonian positivity of massive spin-2 particles via a rank-2 tensor

D Benndorf, D Dalmazi and A L R dos Santos

Massive spin-2 particles via embedment of the Fierz-Pauli equations of motion D Dalmazi, A L R dos Santos and E L Mendonça

Recent developments in bimetric theory Angnis Schmidt-May and Mikael von Strauss

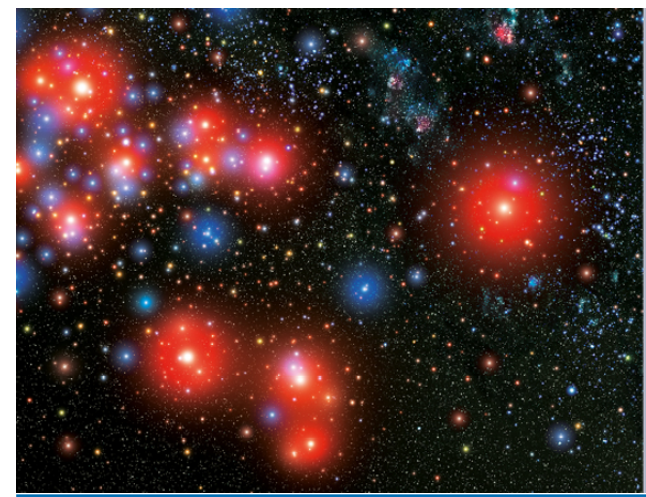

A.A IOP Astronomy ebooks

Part of your publishing universe and your first choice for astronomy, astrophysics, solar physics and planetary science ebooks. iopscience.org/books/aas 


\title{
A note on the ubiquity of the traceless spin connection in dual massive spin-2 theories
}

\author{
D Dalmazi \\ UNESP — Campus de Guaratinguetá—DFQ Avenida Dr. Ariberto Pereira da Cunha, \\ 333 CEP 12516-410-Guaratinguetá-SP-Brazil \\ E-mail: dalmazi@feg.unesp.br
}

Received 30 June 2015, revised 10 December 2015

Accepted for publication 26 January 2016

Published 11 March 2016

\begin{abstract}
There are three families of second-order (in derivatives) models for massive spin-2 particles via a nonsymmetric rank-2 tensor. Each family depends upon an arbitrary real parameter. One of the families includes the usual Fierz-Pauli (FP) model. By starting from a dual formulation of the FP theory in terms of a spin connection it is known that one can derive a fourth-order model for massive 'spin-2' particles in arbitrary dimensions. It is a higher dimensional version of the linearized $3 D$ New Massive Gravity. Here, we show that the same approach applied for the other nonsymmetric rank-2 families leads exactly to the same fourth-order theory. We point out that this is a consequence of the ubiquity of a traceless spin connection in the dual formulation. The Weyl symmetry in the massless limit of one of the rank-2 families is behind the appearance of the traceless spin connection in the dual model. We derive dual maps between correlation functions in the rank-2 (vielbein) and rank-3 (traceless spin connection) dual formulations.
\end{abstract}

Keywords: massive spin-2, massive gravity, Fierz-Pauli

\section{Introduction}

The lack of renormalizability of general relativity (GR) [1, 2] has long ago motivated the addition of higher derivative terms to the Einstein-Hilbert action. It is known [3] however, that higher derivatives of the helicity two modes in $D=3+1$ introduce ghosts at tree level. The case of topologically massive gravity (TMG) [4] in $D=2+1$ shows that higher derivatives may not necessarily lead to ghosts at tree level, at least if we allow the graviton to be massive, which presently has its own motivations. See the review works on massive gravity in [5, 6] and [7]. One might argue that TMG is a very peculiar model which describes only one $(+2$ or -2$)$ helicity state, with parity breaking. Many years later, however, it was shown in [8], still in $D=2+1$, that the addition of quadratic terms in the curvature (parity 
invariant), including higher derivatives of helicity two modes, can still avoid ghosts at tree level and even beyond tree level [9, 10]

Although the New Massive Gravity (NMG) of [8] is probably not renormalizable [11], the work of [8] has raised interest on a possible generalization of NMG to higher dimensions. In [12] a $4 D$ linearized NMG has been suggested. It correctly describes a massive spin-2 particle in $D=4$ and contains a fourth-order term in derivatives, as in the case of the linearized NMG. However, it is much too similar to its $3 D$ counterpart in the sense that the fourth-order term has more local symmetries than the second-order one. Namely, there is a generalized Weyl symmetry in the higher derivative term which means, as pointed out in [13] in the case of NMG, that there are degrees of freedom only present in the second-order term and those will have the usual $1 / p^{2}$ ultraviolet behavior instead of $1 / p^{4}$. The present work emerged as an attempt to overcome such difficulty by generalizing the work [12] and its $D$ dimensional generalization [14].

The works [12] and [14] are based on a first-order formulation of the paradigmatic massive spin-2 theory of Fierz and Pauli (FP) [15] with one extra ingredient. Namely, the FP rank-2 tensor must be nonsymmetric ${ }^{1}: e_{[\mu \nu]} \neq e_{[\nu \mu]}$. The first-order theory used in [12] is formulated in terms of a linearized spin connection $\omega_{\mu \nu \rho}=-\omega_{\mu \rho \nu}$ and a general rank-2 tensor $e_{\mu \nu}$. Schematically, it can be written as

$$
\mathcal{L}^{(1)}[\omega, e]=m^{2} \omega \cdot \omega+m \omega \partial e-m^{2} e \cdot e .
$$

The last term is the usual FP mass term $\left(e_{\mu \nu} e^{\nu \mu}-e^{2}\right)$ while the first is an appropriate spin connection mass term ${ }^{2}$. The Gaussian integral over the spin connection leads to the usual FP theory [15] while the Gaussian integral over $e_{\mu \nu}$ leads to a dual model. Schematically again, the dual rank-3 model can be written as

$$
\mathcal{L}_{\omega, \omega}[\omega]=(\partial \omega)^{2}+m^{2} \omega \cdot \omega .
$$

It turns out, not totally surprisingly ${ }^{3}$, that the kinetic term by itself $(\partial \omega)^{2}$ has no particle content. Consequently, one can build up a master action, similar to formula (3.14) of [12],

$$
\mathcal{L}_{M}[\omega, \Omega]=(\partial \omega)^{2}+m^{2} \omega \cdot \omega-[\partial(\omega-\Omega)]^{2}=m^{2} \omega \cdot \omega-2 \omega \partial^{2} \Omega-\partial \Omega \cdot \partial \Omega
$$

On the one hand, the shift $\Omega \rightarrow \Omega+\omega$ and the absence of particle content of $(\partial \Omega)^{2}$ shows that $\mathcal{L}_{M}[\omega, \Omega]$ has the same content of $\mathcal{L}_{\omega \omega}[\omega]$, i.e., a massive spin-2 particle. On the other hand, the Gaussian integral over $\omega_{\mu \nu \rho}$ gives rise to a fourth-order model $\mathcal{L}[\Omega]$ which by construction describes also a massive spin-2 particle. In $D=3$ it becomes the linearized $\mathrm{NMG}^{4}$ of [8]. A natural nonlinear completion of $\mathcal{L}[\Omega]$ in $D=3$ leads to the full NMG model. In $D=4 \mathcal{L}[\Omega]$ is the $4 D$ NMG of [12] while in $D$-dimensions it is the $D$-dimensional linearized NMG of $[14,18]$. The model of $[12,14,18]$ has a generalized Weyl symmetry in

1 Throughout this work we use $\eta_{\mu \nu}=\operatorname{diag}(-,+, \cdots,+)$ and the notation $e_{[\mu \nu]}=\left(e_{\mu \nu}-e_{\nu \mu}\right) / 2$, $e_{(\mu \nu)}=\left(e_{\mu \nu}+e_{\nu \mu}\right) / 2, e \equiv \eta^{\mu \nu} e_{\mu \nu}$. Although the antisymmetric components $e_{[\mu \nu]}$ vanish on shell, they turn out to be important also to prove duality between the rank-3 massive spin-2 model of Curtright and Freund [16] and the usual FP theory, see [17]

2 Throughout this work the linearized spin connection $\omega_{\mu \nu \alpha}$ has the same mass dimension of the linearized vierbein, in contrast to the usual notation for a spin connection, namely, $\omega_{\mu \nu \alpha}^{\text {ours }}=\omega_{\mu \nu \alpha}^{\text {usual }} / \mathrm{m}$.

3 In higher rank dual models of massive spin-zero and spin-1 particles such as $\left(\partial_{\mu} A^{\mu}\right)^{2}+m^{2} A_{\mu} A^{\mu}$ and $\left(\partial^{\mu} W_{\mu \nu}\right)^{2}+m^{2}\left[W_{\mu \nu}^{2}-W^{2} /(D-1)\right] / 2$ respectively, the kinetic terms have no particle content, see details in [14]. ${ }^{4}$ The connection between $\Omega_{\mu \nu \rho}$ and the metric is made through the $3 \mathrm{D}$ identity $\Omega_{\mu \nu \rho}=\epsilon_{\nu \rho \beta} h_{\mu}{ }_{\mu}$. It turns out that only the traceless piece of $\Omega_{\mu \nu \rho}$ survives. The traceless condition $\eta^{\mu \nu} \Omega_{\mu \nu \rho}=0$ leads to a symmetric rank-2 tensor $h_{\beta \mu}=h_{\mu \beta}$ which is the metric fluctuation, $g_{\mu \nu}=\eta_{\mu \nu}+h_{\mu \nu}$. 
the fourth-order term which is, as already mentioned, an obstacle to building up a renormalizable nonlinear completion.

The fact that $e_{[\mu \nu]} \neq 0$ in (2) opens up new possibilities. In [19-21] one has shown that there are alternative massive spin-2 models to the usual FP theory in $D=4$ if we assume from the start that $e_{[\mu \nu]} \neq 0$. In the present work, we replace the starting point (1) by rather general first-order actions which interpolate between those alternative rank-2 models and a dual rank-3 model. It turns out that the same fourth-order model $\mathcal{L}[\Omega]$ of $[14,18]$ in $D$-dimensions is recovered. We show that the ubiquity of a rank-3 traceless model is behind this fact.

In section 2 we deduce the alternative first-order theories (15) and (19). In section 3 we obtain the corresponding spin connection dual models and demonstrate their reduction to the ubiquitous traceless rank-3 model $\mathcal{L}_{\bar{\Omega}}$, see (28). In section 4 we find dual maps between $\mathcal{L}_{\bar{\Omega}}$ and the alternative rank- 2 models, we also reproduce the FP conditions directly from $\mathcal{L}_{\bar{\Omega}}$ and comment on its massless limit in $D$-dimensions. In section 5 we draw our conclusions. In appendix A we deduce some general formulas regarding the Gaussian integral over the linearized spin connection. In appendix B we deduce, in the simplest way we know, the fourth-order $D$-dimensional NMG model, while in appendix C we prove the conditions (12) for a vector symmetry.

\section{More general first-order massive spin-2 models}

In [20-22] we have shown that there are three independent families of second-order models which describe a massive 'spin-2' particle in $D$ dimensions via an arbitrary (nonsymmetric) rank-2 tensor $e_{\mu \nu}$. They can all be written as

$$
\begin{aligned}
\mathcal{L}\left[e_{\mu \nu}\right]= & a_{1}\left(\partial^{\alpha} e_{\alpha \beta}\right)^{2}+a_{2}\left(\partial^{\alpha} e_{\alpha \nu}\right)\left(\partial_{\beta} e^{\nu \beta}\right)+a_{3}\left(\partial^{\nu} e_{\mu \nu}\right)^{2}+b_{1} e \square e+b_{2} \partial^{\mu} e \partial^{\alpha} e_{\alpha \mu} \\
& +\frac{p_{1}}{2} e_{\mu \nu} \square e^{\mu \nu}+\frac{p_{2}}{2} e_{\mu \nu} \square e^{\nu \mu}-\frac{m^{2}}{2}\left(e_{\mu \nu} e^{\nu \mu}+c_{1} e^{2}+c_{2} e_{[\mu \nu]}^{2}\right) .
\end{aligned}
$$

For all three families we have:

$$
\left(a_{2}, a_{3}, p_{1}, p_{2}\right)=\left(\frac{1}{2}, \frac{1}{4}, \frac{1}{2}, \frac{1}{2}\right) .
$$

Each family contains one free coefficient as shown in the following table.

The sum $S=a_{1}+a_{2}+a_{3}$ is an interesting index since it is invariant under invertible trivial redefinitions $e_{\mu \nu} \rightarrow A e_{\mu \nu}+(1-A) e_{\nu \mu}+B \eta_{\mu \nu} e$, with $(A, B) \neq(1 / 2,-1 / D)$. In the last column, we display the gauge symmetry of the corresponding massless theory.

The first family includes $\left(c_{2}=0\right)$ the usual FP model [15]. In the second, the subscript 'nFP' stands for non-Fierz-Pauli since $c_{1}$ is arbitrary and the mass term does not need to fit the FP fine-tuning $c_{1}=-1$. The third family overlaps the other two at $a_{1}=1 / 4$ (FP case with $\left.c_{2}=0\right)$ and $a_{1}=(3-D) /[4(D-1)]$ (nFP case with $\left.c_{1}=-1\right)$. At these points we have the corresponding enhancement of the local symmetries of the massless theories. Moreover, $\mathcal{L}_{a_{1}}$ at $a_{1}=-1 / 4$ becomes the model suggested in [19]. The three families have been derived in [20] in $D=4$ from the requirement that it contains only one massive pole in the spin-2 sector of the propagator and its residue has the correct sign for a physical particle. From the equations of motion of (4) one derives the Klein-Gordon equation and the FP conditions for all three families, namely 
Table 1. Three families of massive spin-2 models.

\begin{tabular}{lccccccc}
\hline family & $a_{1}$ & $b_{1}$ & $b_{2}$ & $c_{1}$ & $c_{2}$ & $S$ & $\delta e_{\mu \nu}(m=0)$ \\
\hline $\mathcal{L}_{F P}$ & $1 / 4$ & $-1 / 2$ & -1 & -1 & free & 1 & $\partial_{\nu} \xi_{\mu}+\Lambda_{[\mu \nu]}$ \\
$\mathcal{L}_{n F P}$ & $-\frac{D-3}{4(D-1)}$ & $-\frac{1}{2(D-1)}$ & $-\frac{1}{D-1}$ & free & 0 & $\frac{D}{2(D-1)}$ & $\partial_{\nu} \xi_{\mu}+\eta_{\mu \nu} \phi$ \\
$\mathcal{L}_{a_{1}}$ & free & $-\left(a_{1}+\frac{1}{4}\right)$ & $-2\left(a_{1}+\frac{1}{4}\right)$ & -1 & 0 & $a_{1}+\frac{3}{4}$ & $\partial_{\nu} \xi_{\mu}$ \\
\hline
\end{tabular}

$$
\left(\square-m^{2}\right) e_{\mu \nu}=0 ; e_{[\mu \nu]}=0 ; \eta^{\mu \nu} e_{\mu \nu}=e=0 ; \partial^{\nu} e_{\mu \nu}=0
$$

The gauge parameter $\Lambda_{[\mu \nu]}$ is an arbitrary antisymmetric tensor which allows us, together with the arbitrariness of $c_{2}$, to get rid of $e_{[\mu \nu]}$ off-shell for the FP model, which is usually presented in terms of a symmetric tensor $e_{(\mu \nu)}$.

In order to generalize to the second and third families, the usual vielbein-spin connection first-order version of the FP theory as given for instance in [17, 23] we start with a rather general first-order ansatz with nine new coefficients to be determined and take $c_{2}=0$, namely,

$\mathcal{L}_{\omega e}=k \omega_{\alpha \beta \gamma}^{2}+r \omega_{\alpha \beta \gamma} \omega^{\gamma \beta \alpha}+s \omega_{\mu} \omega^{\mu}+2 \omega_{\alpha \beta \gamma} F^{\alpha \beta \gamma}-\frac{m^{2}}{2}\left(e_{\mu \nu} e^{\nu \mu}+c_{1} e^{2}\right)$.

where

$$
\begin{gathered}
\omega_{\alpha \beta \gamma}=-\omega_{\alpha \gamma \beta} ; \quad \omega_{\gamma}=\eta^{\alpha \beta} \omega_{\alpha \beta \gamma}, \\
F_{\alpha \beta \gamma}=a \partial_{\alpha}\left(e_{\beta \gamma}-e_{\gamma \beta}\right)+b\left(\partial_{\beta} e_{\alpha \gamma}-\partial_{\gamma} e_{\alpha \beta}\right)+c\left(\partial_{\beta} e_{\gamma \alpha}-\partial_{\gamma} e_{\beta \alpha}\right) \\
+\eta_{\alpha \beta}\left(d \partial_{\gamma} e+t \partial^{\mu} e_{\mu \gamma}+u \partial^{\mu} e_{\gamma \mu}\right)-\eta_{\alpha \gamma}\left(d \partial_{\beta} e+t \partial^{\mu} e_{\mu \beta}+u \partial^{\mu} e_{\beta \mu}\right)
\end{gathered}
$$

In the appendix A we show that the Gaussian integral over the 'spin-connection' $\omega_{\alpha \beta \gamma}$ gives rise to the second-order Lagrangian:

$$
\begin{aligned}
\mathcal{L}_{e e}= & \frac{1}{(r-k)(r+2 k)}\left[(2 k-r) F_{\alpha \beta \gamma}^{2}-2 r F_{\alpha \beta \gamma} F^{\gamma \beta \alpha}+\frac{4 s(r-k)}{[2 k+r+s(D-1)]} F_{\gamma}^{2}\right] \\
& -\frac{m^{2}}{2}\left(e_{\mu \nu} e^{\nu \mu}+c_{1} e^{2}\right) .
\end{aligned}
$$

In order to avoid constraints on $F_{\alpha \beta \gamma}$ we have assumed :

$$
(r-k)(r+2 k)[2 k+r+s(D-1)] \neq 0 .
$$

Comparing (10) with (4) we obtain complicated formulas for the seven coefficients $a_{i}, b_{j}$, $p_{j}$, with $i=1,2,3$ and $j=1,2$, in terms of the nine parameters $(a, b, c, d, t, u, k, r, s)$ appearing in (7). The explicit formulas are displayed in appendix A. Since they are rather involved, it is convenient to make use of the conditions on the nine parameters of (7) which guarantee the local massless gauge symmetries appearing in table 1. As shown in appendix C, invariance of $\mathcal{L}_{\omega e}(m=0)$ under the ubiquitous vector symmetry $\delta e_{\mu \nu}=\partial_{\nu} \xi_{\mu}$ and the fact that the only consistent massive spin-2 models in terms of $e_{\mu \nu}$ are the ones defined in (5) and table 1 , require that

$$
d+t=0 ; \quad u=0 ; \quad a=c .
$$

From the formulas (86) in appendix B, and, see (5), $p_{+}=\left(p_{1}+p_{2}\right) / 2=1 / 2$ and $p_{-}=\left(p_{1}-p_{2}\right) / 2=0$ together with $a=c$, we find $r$ and $k$ as functions of $b$ and $c$ : 


$$
r=8\left[(b-c)^{2}+2 c^{2}\right] ; \quad k=8 c(2 b-c) .
$$

So far, formulas (12) and (13) hold for both families $\mathcal{L}_{n F P}$ and $\mathcal{L}_{a_{1}}$. Sticking to the second case, plugging (12) and (13) in (81) we obtain $a_{1}=a_{1}(s)$, and inverting such relationship we have:

$$
s=-8 \frac{\left[(b+c)^{2}\left(1+4 a_{1}\right)-4(b+c) d+2(D-1) d^{2}\right]}{4(D-1) a_{1}+D-3} .
$$

Thus, we find the first-order version of the third family $\mathcal{L}_{a_{1}}$ :

$$
\begin{aligned}
\mathcal{L}_{a_{1}}^{(1)}= & k \omega_{\alpha \beta \gamma}^{2}+r \omega_{\alpha \beta \gamma} \omega^{\gamma \beta \alpha}+s \omega_{\mu} \omega^{\mu}-\frac{m^{2}}{2}\left(e_{\mu \nu} e^{\nu \mu}-e^{2}\right) \\
& +4\left[c \omega_{\alpha \beta \gamma}\left(\partial^{\alpha} e^{\beta \gamma}-\partial^{\gamma} e^{\beta \alpha}\right)+b \omega_{\alpha \beta \gamma} \partial^{\beta} e^{\alpha \gamma}+d \omega_{\mu}\left(\partial^{\mu} e-\partial_{\alpha} e^{\alpha \mu}\right)\right] .
\end{aligned}
$$

where $k, r, s$ are given in (13) and (14) while $b, c, d$ remain arbitrary except for the condition :

$$
(b-2 c)(b+c)[b-(D-1) d+c]\left[4(D-1) a_{1}+D-3\right] \neq 0 .
$$

which stems from (11) and the emergence of the Weyl symmetry $\delta_{W} e_{\mu \nu}=\eta_{\mu \nu} \phi$ in the massless theory at $a_{1}=(3-D) /[4(D-1)]$ where the second family $\mathcal{L}_{a_{1}}$ coincides with the third one $\mathcal{L}_{n F P}$. The arbitrariness of $b, c, d$ is a consequence of trivial field redefinitions of the kind:

$$
\omega_{\alpha \beta \gamma} \rightarrow l_{1} \omega_{\alpha \beta \gamma}+l_{2}\left(\omega_{\gamma \beta \alpha}-\omega_{\beta \gamma \alpha}\right)+l_{3}\left(\eta_{\alpha \beta} \omega_{\gamma}-\eta_{\alpha \gamma} \omega_{\beta}\right),
$$

which do not change the result of the Gaussian integral over the 'spin-connection'. The constants $l_{1}, l_{2}, l_{3}$ are arbitrary except for the fact that they must be such that they allow for an inverse transformation.

Likewise, regarding the second family $\mathcal{L}_{n F P}$, the reader can check that the requirement of the Weyl invariance on (7) imposes:

$$
b=u+t+D d-c .
$$

From (12), (13) and (18) we deduce the first-order nFP model:

$$
\begin{aligned}
\mathcal{L}_{n F P}^{(1)}= & k \omega_{\alpha \beta \gamma}^{2}+r \omega_{\alpha \beta \gamma} \omega^{\gamma \beta \alpha}+s \omega_{\mu} \omega^{\mu}-\frac{m^{2}}{2}\left(e_{\mu \nu} e^{\nu \mu}+c_{1} e^{2}\right) \\
& +4\left[c \omega_{\alpha \beta \gamma}\left(\partial^{\alpha} e^{\beta \gamma}-\partial^{\beta} e^{\alpha \gamma}+\partial^{\beta} e^{\gamma \alpha}\right)\right. \\
& \left.+d(D-1) \omega_{\alpha \beta \gamma} \partial^{\beta} e^{\alpha \gamma}+d \omega_{\mu}\left(\partial^{\mu} e-\partial_{\alpha} e^{\alpha \mu}\right)\right] .
\end{aligned}
$$

where

$$
r=8\left\{[d(D-1)-2 c]^{2}+2 c^{2}\right\} ; k=-8 c[3 c-2 d(D-1)] .
$$

Due to the field redefinitions (17), the parameters $c, d, s$ remain arbitrary except for the condition (11) which now becomes

$$
d[3 c-2 d(D-1)]\left[s+8 d^{2}(D-1)\right] \neq 0 .
$$

In the next section, we obtain from (15) and (19) dual models $\mathcal{L}_{a_{1}}^{*}$ and $\mathcal{L}_{n F P}^{*}$ in terms of a rank-3 field $\omega_{\alpha \beta \gamma}$. 


\section{Dual rank-3 model}

Now, we Gaussian integrate over $e_{\mu \nu}$ in the first-order theories (15) and (19) in order to obtain dual models. It is convenient to begin with the nFP model (19). The action (19) can be written as

$$
\begin{aligned}
\mathcal{L}_{n F P}^{(1)}= & k \omega_{\alpha \beta \gamma}^{2}+r \omega_{\alpha \beta \gamma} \omega^{\gamma \beta \alpha}+s \omega_{\mu} \omega^{\mu}-\frac{m^{2}}{2}\left[\left(e_{\mu \nu}-\bar{f}_{\mu \nu}\right)\left(e^{\nu \mu}-\bar{f}^{\nu \mu}\right)+c_{1} e^{2}\right] \\
& +\frac{m^{2}}{2} \bar{f}_{\nu \mu} \bar{f}^{\mu \nu} .
\end{aligned}
$$

where we have introduced the traceless tensor $\bar{f}_{\nu \mu}$ :

$\bar{f}_{\nu \mu}=-\frac{4}{m^{2}}\left\{c \partial^{\alpha} \omega_{\alpha \mu \nu}+[c-(D-1) d] \partial^{\gamma} \omega_{\mu \nu \gamma}-c \partial^{\gamma} \omega_{\nu \mu \gamma}+d\left(\eta_{\mu \nu} \partial^{\beta} \omega_{\beta}-\partial_{\mu} \omega_{\nu}\right)\right\}$.

Remarkably, $\bar{f}_{\mu \nu}$ can be written as

$$
\bar{f}_{\mu \nu}=\frac{\partial^{\gamma} \bar{\Omega}_{\mu \nu \gamma}}{m},
$$

where we have the traceless $\left(\eta^{\mu \nu} \bar{\Omega}_{\mu \nu \gamma}=0\right)$ 'spin-connection':

$\bar{\Omega}_{\mu \nu \gamma}=\frac{4}{m}\left[d(D-1) \omega_{\mu \nu \gamma}+d\left(\eta_{\mu \nu} \omega_{\gamma}-\eta_{\mu \gamma} \omega_{\nu}\right)+c\left(\omega_{\nu \mu \gamma}-\omega_{\mu \nu \gamma}-\omega_{\gamma \mu \nu}\right)\right]$.

The coupling between the field $e_{\mu \nu}$ and a traceless tensor like $\bar{f}_{\nu \mu}$ in (19) is a consequence of the Weyl symmetry $\left(\delta_{W} e_{\mu \nu}=\eta_{\mu \nu} \phi\right)$. After the shift $e_{\mu \nu} \rightarrow \tilde{e}_{\mu \nu}+\bar{f}_{\mu \nu}$ in (22) and neglecting the quadratic term in the decoupled field $\tilde{e}_{\mu \nu}$ we obtain after some algebra

$$
\begin{aligned}
& \mathcal{L}_{n F P}^{*}=\frac{m^{2}}{2} \bar{f}_{\nu \mu} \bar{f}^{\mu \nu}+k \omega_{\alpha \beta \gamma}^{2}+r \omega_{\alpha \beta \gamma} \omega^{\gamma \beta \alpha}+s \omega_{\mu} \omega^{\mu}, \\
& =\frac{1}{2} \partial^{\gamma} \bar{\Omega}_{\mu \nu \gamma} \partial_{\gamma} \bar{\Omega}^{\nu \mu \gamma}+\frac{m^{2}}{2} \bar{\Omega}_{\alpha \beta \gamma} \bar{\Omega}^{\gamma \beta \alpha}+\left[s+d^{2}(D-1)\right] \omega_{\mu}^{2} .
\end{aligned}
$$

Dropping the decoupled ${ }^{5}$, pure trace $\omega_{\mu}^{2}$ term we have the $\bar{\Omega}$-model:

$$
\mathcal{L}_{\bar{\Omega}}=\frac{1}{2} \partial^{\gamma} \bar{\Omega}_{\mu \nu \gamma} \partial_{\gamma} \bar{\Omega}^{\nu \mu \gamma}+\frac{m^{2}}{2} \bar{\Omega}_{\alpha \beta \gamma} \bar{\Omega}^{\gamma \beta \alpha} .
$$

The model (28) is probably the most compact way of writing down a Lorentz covariant Lagrangian density for a massive 'spin-2' particle in arbitrary dimensions. It has appeared before in [17] as a dual theory to the usual FP model (first family of table 1). As shown in [17] it is equivalent to the model of [16] in $D=4$. It is not the most economical description of massive spin-2 particle in general, since $\bar{\Omega}_{\mu \nu \rho}$ contains $D^{2}(D-1) / 2-D$ components which are, in $D \geqslant 4$, larger than the number of components of a symmetric rank-2 tensor (FP theory): $D(D+1) / 2$. They match only in $D=3$ where, see [14], we can always write $\bar{\Omega}_{\mu \nu \rho}=\epsilon_{\nu \rho \alpha} e_{\mu}^{\alpha}$ and the traceless condition implies $e_{\mu \nu}=e_{\nu \mu}$.

We point out that the existence of the family $\mathcal{L}_{n F P}$ with Weyl symmetry in the massless limit makes the appearance of a traceless tensor $\bar{\Omega}_{\mu \nu \rho}$ quite natural.

5 Note that the right-hand side of (25) is invariant under $\delta \omega_{\mu \nu \gamma}=\eta_{\mu \nu} \phi_{\gamma}-\eta_{\mu \gamma} \phi_{\nu}$ which means invariance under an arbitrary shift of the trace $\delta \omega_{\mu}=(D-1) \phi_{\mu}$. Therefore, the trace $\omega_{\mu}$ is effectively not present in the first two terms of (27). 
Moving now to the dual of the third family $\mathcal{L}_{a_{1}}$, similar to (26), we notice that Gaussian integrating over $e_{\mu \nu}$ in (15) we obtain

$$
\mathcal{L}_{a_{1}}^{*}=k \omega_{\alpha \beta \gamma}^{2}+r \omega_{\alpha \beta \gamma} \omega^{\gamma \beta \alpha}+s \omega_{\mu} \omega^{\mu}+\frac{m^{2}}{2}\left(f_{\nu \mu} f^{\mu \nu}-f^{2}\right) .
$$

where

$$
f_{\mu \nu}=\frac{\partial^{\gamma} \tilde{\bar{\Omega}}_{\mu \nu \gamma}}{m}+\frac{4}{m^{2}}\left(d-\frac{b+c}{D-1}\right) \partial_{\nu} \omega_{\mu}
$$

Another traceless 'spin-connection' has been introduced

$$
\tilde{\Omega}_{\nu \mu \gamma}=\frac{4}{m}\left[c\left(\omega_{\mu \nu \gamma}-\omega_{\gamma \nu \mu}\right)+b \omega_{\nu \mu \gamma}+\frac{(b+c)}{D-1}\left(\eta_{\nu \gamma} \omega_{\mu}-\eta_{\mu \nu} \omega_{\gamma}\right)\right] \text {. }
$$

Plugging back in $\mathcal{L}_{a_{1}}^{*}$ and neglecting once again a term proportional to $\omega_{\mu}^{2}$ we have exactly the same result (28) with $\bar{\Omega}_{\mu \nu \gamma}$ replaced by $\tilde{\bar{\Omega}}_{\mu \nu \gamma}$. For completeness we mention that, as shown in [17], the dual of the first family of table 1 (usual FP model) is once again the $\bar{\Omega}$-model (28) where

$$
\bar{\Omega}_{\mu \nu \gamma} \rightarrow \omega_{\mu \nu \gamma}+\left(\eta_{\mu \gamma} \omega_{\nu}-\eta_{\mu \nu} \omega_{\gamma}\right) /(D-1)
$$

Therefore, all differences among the three families in table 1 disappear in the universal dual model (28). In particular, all dependence on the free parameters $a_{1}, c_{1}$ has been washed out.

\section{More on the dual $\bar{\Omega}$-model}

The universality of the $\bar{\Omega}$-model impels us to study it in more detail. First, we have found it instructive to derive the Klein-Gordon equation and the FP conditions for a massive spin-2 particle directly from (28), different from [17] where it is derived from another model dual to (28) with many more space-time indices. Second, we look at its detailed relationship with the rank-2 models of table 1 and finally we comment on its massless limit.

\subsection{Fierz-Pauli conditions}

We start with the equations of motion ${ }^{6}$ of (28):

$$
K^{\alpha \beta \gamma} \equiv \frac{\delta S_{\bar{\Omega}}}{\delta \bar{\Omega}_{\alpha \beta \gamma}}=\partial^{\gamma} \partial_{\rho} \bar{\Omega}^{\beta \alpha \rho}-\partial^{\beta} \partial_{\rho} \bar{\Omega}^{\gamma \alpha \rho}-m^{2}\left(\bar{\Omega}^{\gamma \beta \alpha}-\bar{\Omega}^{\beta \gamma \alpha}\right)=0 .
$$

The kinetic term of $S_{\bar{\Omega}}$ is invariant under:

$$
\delta_{\Lambda} \bar{\Omega}_{\alpha \beta \gamma}=\partial^{\mu} \Lambda_{[\mu \beta \gamma] \alpha}+\frac{1}{D-1}\left[\eta_{\alpha \beta} \partial^{\mu} \Lambda_{[\mu \rho \gamma]}^{\rho}-\eta_{\alpha \gamma} \partial^{\mu} \Lambda_{[\mu \rho \beta]}^{\rho}\right] .
$$

where $\Lambda_{[\mu \beta \gamma] \alpha}$ is totally antisymmetric in the first three indices. Motivated by the symmetry (34) we look at the following cyclic combination of the equations of motion:

$$
\partial^{\mu} K^{\alpha \beta \gamma}+\partial^{\gamma} K^{\alpha \mu \beta}+\partial^{\beta} K^{\alpha \gamma \mu}=0 .
$$

\footnotetext{
${ }^{6} S_{\bar{\Omega}}$ is the action corresponding to the Lagrangian density $\mathcal{L}_{\bar{\Omega}}$ in (28).
} 
which leads to the constraints

$$
\partial^{\mu} F^{[\gamma \beta] \alpha}+\partial^{\gamma} F^{[\beta \mu] \alpha}+\partial^{\beta} F^{[\mu \gamma] \alpha}=0 .
$$

where $F^{[\gamma \beta] \alpha}=\bar{\Omega}^{\gamma \beta \alpha}-\bar{\Omega}^{\beta \gamma \alpha}$. From the general solution of (36) a rank-2 tensor arises:

$$
\bar{\Omega}^{\gamma \beta \alpha}-\bar{\Omega}^{\beta \gamma \alpha}=\partial^{\gamma} \tilde{\boldsymbol{e}}^{\beta \alpha}-\partial^{\beta} \tilde{\boldsymbol{e}}^{\gamma \alpha} .
$$

The tensor $\tilde{e}^{\mu \nu}$ is defined up to a gauge transformation $\delta \tilde{e}^{\mu \nu}=\partial^{\mu} \phi^{\nu}$. From the trace of (37) we have

$$
\partial_{\mu} \tilde{e}^{\gamma \mu}=\partial^{\gamma} \tilde{e} .
$$

From (37) and (38) we deduce

$$
\partial_{\alpha}\left(\bar{\Omega}^{\gamma \beta \alpha}-\bar{\Omega}^{\beta \gamma \alpha}\right)=0 .
$$

From (37) back in the equations of motion (33) we have

$$
\partial^{\gamma}\left(\partial_{\rho} \bar{\Omega}^{\beta \alpha \rho}-m^{2} \tilde{\boldsymbol{e}}^{\beta \alpha}\right)-\partial^{\beta}\left(\partial_{\rho} \bar{\Omega}^{\gamma \alpha \rho}-m^{2} \tilde{\boldsymbol{e}}^{\gamma \alpha}\right)=0 .
$$

Whose general solution gives us:

$$
\partial_{\rho} \bar{\Omega}^{\beta \alpha \rho}=m^{2} \tilde{e}^{\beta \alpha}+\partial^{\beta} A^{\alpha} \equiv m^{2} e^{\beta \alpha} .
$$

From the trace of (41) we have the first of the FP conditions:

$$
\eta_{\alpha \beta} e^{\beta \alpha}=e=0 \text {. }
$$

Due to (42), the otherwise arbitrary vector field $A_{\mu}$ must satisfy

$$
\partial \cdot A=-m^{2} \tilde{e} .
$$

From (41) in (39) and $\partial_{\alpha}$ on (41) we have the remaining FP conditions:

$$
\begin{aligned}
& e^{[\alpha \beta]}=0 . \\
& \partial_{\alpha} e^{\beta \alpha}=0,
\end{aligned}
$$

Adding up cyclic permutations of (37), using (41) and (44) we have

$$
2\left(\bar{\Omega}^{\gamma \beta \alpha}+\bar{\Omega}^{\alpha \gamma \beta}+\bar{\Omega}^{\beta \alpha \gamma}\right)=\partial^{\gamma} e^{[\beta \alpha]}+\partial^{\beta} e^{[\alpha \gamma]}+\partial^{\alpha} e^{[\gamma \beta]}=0 .
$$

Thus,

$$
\bar{\Omega}^{\alpha \beta \gamma}=\bar{\Omega}^{\gamma \beta \alpha}-\bar{\Omega}^{\beta \gamma \alpha}=\partial^{\gamma} e^{\beta \alpha}-\partial^{\beta} e^{\gamma \alpha} .
$$

Back in the general solution (41) of the equations of motion (33) and using (45) we finally obtain the Klein-Gordon equations:

$$
\left(\square-m^{2}\right) e^{\mu \nu}=0 .
$$

In summary, the general solution to the original equations of motion (33) is given by (47) where $e^{\mu \nu}$ satisfies the FP conditions (42), (45), (44) and the Klein-Gordon equation. This corresponds to a massive 'spin-2' particle and nothing else.

\subsection{Dual maps}

Now, we turn to the dual maps which relate correlation functions in the families of table 1 and the common dual $\bar{\Omega}$-model. We start with the simplest case, i.e., the duality between $\mathcal{L}_{n F P}$ and $\mathcal{L}_{\bar{\Omega}}$, 


$$
\begin{aligned}
& W[J, \bar{J}]=\int \mathcal{D} \omega_{\mu \nu \alpha} \mathcal{D} e_{\mu \nu} e^{i \int d^{D} x\left[\mathcal{L}_{n F P}^{(1)}+e_{\mu \nu} J^{\mu \nu}+\bar{\Omega}_{\mu \nu \alpha} \bar{J}^{\mu \nu \alpha}\right]} \\
& =\int \mathcal{D} \bar{\Omega}_{\mu \nu \alpha} \mathcal{D} e_{\mu \nu} e^{i \int d^{D} x\left[\mathcal{L}_{M}+e_{\mu \nu} J^{\mu \nu}+\bar{\Omega}_{\mu \nu \alpha} \bar{J}^{\mu \nu \alpha}\right]}
\end{aligned}
$$

where the master Lagrangian [24] $\mathcal{L}_{M}$ is given by

$$
\mathcal{L}_{M}\left[e_{\mu \nu}, \bar{\Omega}_{\mu \nu \alpha}\right]=\frac{m^{2}}{2} \bar{\Omega}_{\alpha \beta \gamma} \bar{\Omega}^{\gamma \beta \alpha}+m e^{\alpha \beta} \partial^{\gamma} \bar{\Omega}_{\alpha \beta \gamma}-\frac{m^{2}}{2}\left(e_{\mu \nu} e^{\nu \mu}-e^{2}\right),
$$

In (49) we have split $\mathcal{D} \omega_{\mu \nu \rho}=\mathcal{D} \bar{\Omega}_{\mu \nu \rho} \mathcal{D} \omega_{\mu}$ and integrated over $\mathcal{D} \omega_{\mu}$. The Lagrangian $\mathcal{L}_{n F P}^{(1)}$ given in (19) differs from $\mathcal{L}_{M}$ by a pure trace term proportional to $\omega_{\mu}^{2}$, so the integral over $\omega_{\mu}$ amounts to an overall constant that we have dropped. Starting from (50) we either integrate over $e_{\mu \nu}$ or $\bar{\Omega}_{\mu \nu \rho}$. The comparison between these two routes leads to the dual maps below. Integrating over $e_{\mu \nu}$ we have up to an overall constant,

$$
\begin{aligned}
W[J, \bar{J}]= & \int \mathcal{D} \bar{\Omega}_{\mu \nu \alpha} \mathcal{D} e_{\mu \nu} e^{i \int d^{D} x}\left\{\mathcal{L}_{\Omega}-\frac{m^{2}}{2}\left[\left(e_{\mu \nu}-\bar{f}_{\mu \nu}\right)\left(e^{\nu \mu}-\bar{f}_{\nu \mu}\right)-e^{2}\right]+e_{\mu \nu} J^{\mu \nu}+\bar{\Omega}_{\mu \nu \alpha} \bar{J}^{\mu \nu \alpha}\right\} \\
& =\int \mathcal{D} \bar{\Omega}_{\mu \nu \alpha} e^{i \int d^{D} x\left\{\mathcal{L}_{\Omega}+\bar{f}_{\mu \nu} J^{\mu \nu}+\bar{\Omega}_{\mu \nu \alpha} \bar{J}^{\mu \nu \alpha}+\mathcal{O}\left(J^{2}\right)\right\}} .
\end{aligned}
$$

The traceless tensor $\bar{f}_{\mu \nu}$ is defined in (24). On the other hand, $\mathcal{L}_{M}$ can be written as

$$
\begin{aligned}
\mathcal{L}_{M} & =\frac{m^{2}}{2} \bar{\Omega}_{\alpha \beta \gamma} \bar{\Omega}^{\gamma \beta \alpha}+m \bar{\Omega}_{\alpha \beta \gamma} F^{\alpha \beta \gamma}-\frac{m^{2}}{2}\left(e_{\mu \nu} e^{\nu \mu}-e^{2}\right) \\
& =\frac{1}{2}\left(m \bar{\Omega}_{\alpha \beta \gamma}+F_{\alpha \beta \gamma}+F_{\gamma \beta \alpha}+F_{\beta \alpha \gamma}\right)\left(m \bar{\Omega}^{\gamma \beta \alpha}+F^{\gamma \beta \alpha}+F^{\alpha \beta \gamma}+F^{\beta \gamma \alpha}\right)+\mathcal{L}_{n F P}\left[e_{\mu \nu}\right] .
\end{aligned}
$$

where

$$
F^{\alpha \beta \gamma}=\frac{1}{2}\left(\partial^{\beta} e^{\alpha \gamma}-\partial^{\gamma} e^{\alpha \beta}+\frac{\eta^{\alpha \beta} V^{\gamma}-\eta^{\alpha \gamma} V^{\beta}}{D-1}\right) ; \quad V^{\gamma}=\partial^{\gamma} e-\partial_{\mu} e^{\mu \gamma} .
$$

Thus, if we first integrate over $\bar{\Omega}_{\mu \nu \alpha}$ in the generating function we obtain, after the shift $\bar{\Omega}_{\alpha \beta \gamma} \rightarrow \bar{\Omega}_{\alpha \beta \gamma}-\left(F_{\alpha \beta \gamma}+F_{\gamma \beta \alpha}+F_{\beta \alpha \gamma}\right) / m$, the $n F P$ model at $c_{1}=-1$. Up to an overall constant we have:

$$
W[J, \bar{J}]=\int \mathcal{D} e_{\mu \nu} e^{i \int d^{D} x\left[\mathcal{L}_{n F P}+e_{\mu \nu} J^{\mu \nu}+G_{\mu \nu \alpha} \bar{J}^{\mu \nu \alpha}+\mathcal{O}\left(\bar{J}^{2}\right)\right]}
$$

where

$$
\begin{aligned}
G_{\alpha \beta \gamma}[e] & =\left(F_{\alpha \beta \gamma}+F_{\gamma \beta \alpha}+F_{\beta \alpha \gamma}\right) / m \\
& =\left(\partial_{\gamma} e_{(\alpha \beta)}-\partial_{\beta} e_{(\alpha \gamma)}-\partial_{\alpha} e_{[\beta \gamma]}+\frac{\eta^{\alpha \gamma} V^{\beta}-\eta^{\alpha \beta} V^{\gamma}}{D-1}\right) / m .
\end{aligned}
$$

Comparing (52) and (55) we have the maps between correlation functions

$$
\left\langle e_{\mu_{1} \nu_{1}}\left(x_{1}\right) \cdots e_{\mu_{N} \nu_{N}}\left(x_{N}\right)\right\rangle_{n F P}=\left\langle\bar{f}_{\mu_{1} \nu_{1}}\left(x_{1}\right) \cdots \bar{f}_{\mu_{N} \nu_{N}}\left(x_{N}\right)\right\rangle_{\bar{\Omega}}+\text { contact terms, }
$$

$\left\langle\bar{\Omega}_{\mu_{1} \nu_{1} \alpha_{1}}\left(x_{1}\right) \cdots \bar{\Omega}_{\mu_{N} \nu_{N} \alpha_{N}}\left(x_{N}\right)\right\rangle_{\bar{\Omega}}=\left\langle G_{\mu_{1} \nu_{1} \alpha_{1}}\left(x_{1}\right) \cdots G_{\mu_{N} \nu_{N} \alpha_{N}}\left(x_{N}\right)\right\rangle_{n F P}+$ contact terms,

where $\langle\cdots\rangle_{\bar{\Omega}}$ stand for correlation functions in the dual $\bar{\Omega}$-model defined in (28). The contact terms are due to the quadratic terms in the sources in (52) and (55).

Regarding the other two families $\mathcal{L}_{F P}$ and $\mathcal{L}_{a_{1}}$, although it is not natural to use a traceless 'spin connection' $\bar{\Omega}_{\mu \nu \alpha}$, it turns out that the maps (57) and (58) still hold with the replacement 
of $\mathcal{L}_{n F P}$ by $\mathcal{L}_{F P}$ and $\mathcal{L}_{a_{1}}$ and the replacement of $\bar{\Omega}_{\mu \nu \rho}$ by the right-hand side of (32) and $\tilde{\bar{\Omega}}_{\mu \nu \rho}$ given in (31), respectively. In order to make this point clear, we take the first-order FP theory. It consists of the linearized Einstein-Hilbert action written in terms of linearized vielbein and spin-connection, plus the FP mass term,

$\mathcal{L}_{F P}^{(1)}=2\left(\omega_{\alpha \beta \gamma} \omega^{\gamma \beta \alpha}-\omega_{\mu} \omega^{\mu}\right)+2 \omega_{\alpha \beta \gamma} \partial^{\beta} e^{\alpha \gamma}+2 \omega_{\mu}\left(\partial^{\mu} e-\partial_{\alpha} e^{\alpha \mu}\right)-\frac{m^{2}}{2}\left(e_{\mu \nu} e^{\nu \mu}-e^{2}\right)$.

In the corresponding action $S_{F P}^{(1)}$ we can integrate by parts and using the traceless 'spin connection' $\bar{\Omega}_{\mu \nu \rho}$ on the right-hand side of (32) we can write:

$$
\begin{aligned}
\mathcal{L}_{F P}^{(1)}= & \frac{m^{2}}{2} \bar{\Omega}_{\alpha \beta \gamma} \bar{\Omega}^{\gamma \beta \alpha}+m e^{\alpha \beta} \partial^{\gamma} \bar{\Omega}_{\alpha \beta \gamma}-\frac{m^{2}}{2}\left(e_{\mu \nu} e^{\nu \mu}-e^{2}\right) \\
& +\frac{2(D-2)}{D-1} \omega_{\mu}\left(\partial^{\mu} e-\partial_{\alpha} e^{\alpha \mu}\right)-\frac{2(D-1)}{D-1} \omega_{\mu}^{2} .
\end{aligned}
$$

Due to the lack of Weyl symmetry in the massless theory, the trace $\omega_{\mu}$ is nontrivially coupled to $e_{\mu \nu}$. However, the coupling can be undone by the shift $e_{\mu \nu} \rightarrow e_{\mu \nu}+K \partial_{\nu} \omega_{\mu}$ with $K=2(D-2) /\left[m^{2}(D-2)\right]$. Thus, in the path integral we have, up to overall constants,

$$
\begin{gathered}
W[J, \bar{J}]=\int \mathcal{D} \omega_{\mu \nu \alpha} \mathcal{D} e_{\mu \nu} e^{i \int d^{D} x\left[\mathcal{L}_{F P}^{(1)}+e_{\mu \nu} J^{\mu \nu}+\bar{\Omega}_{\mu \nu \alpha} \bar{J}^{\mu \nu \alpha}\right]} \\
=\int \mathcal{D} \bar{\Omega}_{\mu \nu \alpha} \mathcal{D} e_{\mu \nu} \mathcal{D} \omega_{\mu} e^{i \int d^{D} x\left[\mathcal{L}_{M}+\frac{2(D-2)}{D-1} \omega_{\mu}\left(\partial^{\mu} e-\partial_{\alpha} e^{\alpha \mu}\right)-\frac{2(D-1)}{D-1} \omega_{\mu}^{2}+e_{\mu \nu} J^{\mu \nu}+\bar{\Omega}_{\mu \nu \alpha} \bar{J}^{\mu \nu \alpha}\right]} \\
=\int \mathcal{D} \bar{\Omega}_{\mu \nu \alpha} \mathcal{D} e_{\mu \nu} \mathcal{D} \omega_{\mu} e^{i \int d^{D} x\left[\mathcal{L}_{M}-\frac{2(D-1)}{D-1} \omega_{\mu}^{2}-K \omega_{\mu} \partial_{\nu} J^{\mu \nu}+e_{\mu \nu} J^{\mu \nu}+\bar{\Omega}_{\mu \nu \alpha} \bar{J}^{\mu \nu \alpha}\right]} \\
=\int \mathcal{D} \bar{\Omega}_{\mu \nu \alpha} e^{i \int d^{D} x\left\{\mathcal{L}_{\Omega}+\bar{f}_{\mu \nu} J^{\mu \nu}+\bar{\Omega}_{\mu \nu \alpha} \bar{J}^{\mu \nu \alpha}+\mathcal{O}\left(J^{2}\right)\right\}}
\end{gathered}
$$

Instead, if we start from the second line (62) and integrate over $\bar{\Omega}_{\mu \nu \alpha}$ and $\omega_{\mu}$ we end up with

$$
W[J, \bar{J}]=\int \mathcal{D} e_{\mu \nu} e^{i \int d^{D} x\left[\mathcal{L}_{F P}[e]+e_{\mu \nu} J^{\mu \nu}+\bar{G}_{\mu \nu \alpha} \bar{J}^{\mu \nu \alpha}+\mathcal{O}\left(\bar{J}^{2}\right)\right]}
$$

Comparing (64) and (65) we confirm that the dual maps (57) and (58) hold also for the duality between $\mathcal{L}_{\bar{\Omega}}$ and $\mathcal{L}_{F P}$. The case of the third family $\mathcal{L}_{a_{1}}$ is totally analogous to the FP case.

\subsection{The massless limit}

Probably the main motivation for studying massive spin-2 particles is massive gravity [5, 6]. If the graviton has a mass it must be a tiny one. So it is important to look at the massless limit of the massive spin-2 $\bar{\Omega}$-model (28).

The massless limit of (28) can be written in a first order form with the help of a traceless nonsymmetric tensor $\bar{e}_{\mu \nu}$ :

$$
\mathcal{L}_{\bar{\Omega}}(m=0)=\bar{e}_{\mu \nu} \partial_{\rho} \bar{\Omega}^{\mu \nu \rho}-\frac{1}{2} \bar{e}_{\mu \nu} \bar{e}^{\nu \mu}
$$

In the path integral, the integral over $\bar{e}_{\mu \nu}$ leads to the kinetic term of (28) while integrating over $\bar{\Omega}_{\mu \nu \rho}$ we have a functional delta function enforcing the constraint: 


$$
\partial_{\rho} \bar{e}_{\mu \nu}-\partial_{\nu} \bar{e}_{\mu \rho}+\frac{\eta_{\mu \rho} \partial^{\beta} \bar{e}_{\beta \nu}-\eta_{\mu \nu} \partial^{\beta} \bar{e}_{\beta \rho}}{1-D}=0 .
$$

By applying $\partial^{\mu}$ on (67) we have

$$
\partial_{\rho}\left(\partial^{\mu} \bar{e}_{\mu \nu}\right)-\partial_{\nu}\left(\partial^{\mu} \bar{e}_{\mu \rho}\right)=0 .
$$

whose general solution is $\partial^{\mu} \bar{e}_{\mu \nu}=\partial_{\nu} \phi$. Back in (67) we have

$$
\partial_{\rho} f_{\mu \nu}-\partial_{\nu} f_{\mu \rho}=0 \text {. }
$$

where $f_{\mu \nu}=\bar{e}_{\mu}+\eta_{\mu \nu} \phi /(D-1)$. The general solution to (69) introduces an arbitrary vector field: $f_{\mu \nu}=\partial_{\nu} \Phi_{\mu}$. Taking the trace we have $\phi=(D-1) \partial_{\mu} \Phi^{\mu} / D$ and $\bar{e}_{\mu \nu}=\partial_{\nu} \Phi_{\mu}-\eta_{\mu \nu} \partial \cdot \Phi / D$. Finally, back in the last term of (66) we deduce an equivalent theory

$$
\mathcal{L}_{\bar{\Omega}}(m=0) \leftrightarrow-\frac{(D-1)}{2 D}\left(\partial_{\mu} \Phi^{\mu}\right)^{2}
$$

It is known that $\left(\partial_{\mu} \Phi^{\mu}\right)^{2}$, and consequently $\mathcal{L}_{\bar{\Omega}}(m=0)$, is a pure gauge theory with no particle content, see, for instance, [14]. Indeed, as shown in [17], $\mathcal{L}_{\bar{\Omega}}$ is equivalent to the Curtright-Freund theory in $D=4$ which is known to have no particle content [16] at $m=0$. Our demonstration holds in arbitrary dimensions. Thus, by taking the massless limit in (28) we go from $\left(D^{2}-D-2\right) / 2$ degrees of freedom of the massive 'spin-2' particle to zero. This is analogous to other higher rank descriptions of massive particles like, e.g., $\mathcal{L}=\left(\partial_{\mu} A^{\mu}\right)^{2}+m^{2} A_{\mu} A^{\mu}$ which describes a massive scalar, but whose massless limit has no particle content.

Since the kinetic term of (28) has no particle content it can be used as a mixing term in the master action approach of [24] in order to generate a higher-order dual model. In appendix B we confirm that a simple master action in terms of traceless dual fields $\bar{\Omega}_{\mu \nu \rho}$ and $\bar{\omega}_{\mu \nu \rho}$ leads to the same linearized fourth-order theories of $[8,12]$ and $[14,18]$ in $D=2+1$, $D=3+1$ and arbitrary $D$-dimensions, respectively.

\section{Conclusion}

It would certainly be welcome to have a unitary and renormalizable local quantum field theory for gravity (massless gravitons). However, this does not seem to be possible even in the lower dimensional 3D case with massive gravitons.

Although the topologically massive gravity of [4] and the NMG of [8] both have higher derivatives in the Lagrangian, not all degrees of freedom of the metric are present in the higher derivative term due to an increased local symmetry in the higher derivative term compared to the lower second-order one, as pointed out in [13]. The question of finding a new higher-order massive spin-2 theory arises, where both higher and lower derivative terms have the same set of local symmetries. Usually, the higher derivative term has more local symmetries than the mass term, as in the Maxwell-Proca theory, in order to produce the necessary constraints to decrease the exceeding number of components of the tensor fields to the required number of propagating degrees of freedom, like $2 s+1$ for spin-s in $D=3+1$. However, at least in $D=2+1$ there are exceptions, like the massive spin-1 MaxwellChern-Simons theory [4] and the massive spin-2 higher derivative TMG of [25, 26] where both lower and higher derivative terms have the same set of local symmetries.

Here, we have tried to obtain new higher derivative massive 'spin-2' models, in arbitrary dimensions, by generalizing the procedure of [12] where the authors have deduced a fourth- 
order description of massive spin-2 particles in $D=3+1$, still with unbalanced local symmetries in lower and higher derivative terms. The work [12] is based on a first-order frame-like description of the usual FP theory, where the rank-2 tensor (linearized vierbein) is not symmetric $e_{\mu \nu} \neq e_{\nu \mu}$.

In $[20,22]$ we have shown that there are three independent families of second-order theories for a nonsymmetric rank-2 tensor which describe massive spin- 2 particles. The FP family is just one of them, see table 1 in the introduction. So we have generalized [12] for the second and the third family of table 1 . It turns out that the final fourth-order massive spin-2 model is the same one obtained in [12] in $D=3+1$ and in [14] in $D$-dimensions. Here, we have shown that this is a consequence of the ubiquity of the traceless spin connection dual formulation (28). The free parameters of the three independent rank-2 families appearing in table 1 disappear in the dual $\bar{\Omega}$-model (28). From this point of view, it is tempting to think of the $\bar{\Omega}$-model, which has no free parameter, as a more fundamental description of massive spin-2 particles than the usual description in terms of a rank-2 tensor.

To the best of our knowledge, the $\bar{\Omega}$-model has first been found in [17] and it is probably the most compact way of writing down an action for a free massive 'spin-2' particle in arbitrary dimensions. In the last section, we have worked out the FP conditions and analyzed its massless limit in arbitrary dimensions. We have also deduced dual maps between correlation functions in the $\bar{\Omega}$-model and in the corresponding rank- 2 families. The work done here can also be understood as an independent proof of duality between the three families of nonsymmetric rank- 2 tensors since they are all dual to the same $\bar{\Omega}$-model.

The model $\mathcal{L}_{n F P}$, the second family in table 1 , possesses Weyl symmetry in the $m=0$. As we have shown, this makes more natural the appearance of a traceless spin connection.

We note that we could have added further terms including the Levi-Civita tensor, like $\epsilon^{\mu \nu \rho \beta} \omega_{\alpha \rho \beta} \partial^{\alpha} e_{\mu \nu}$ to our first-order frame-like theories in $D=3+1$ but we do not expect new dual $\bar{\Omega}$-models.

Finally, since at linearized level there is no difference between Lorentz and space-time indices, the 'spin-connection' could also be interpreted as the torsion tensor. So (28) would be a pure (traceless) torsion description of massive spin-2 particles.

\section{Acknowledgments}

The work of D D is supported by CNPq (307278/2013-1) and FAPESP (2013/00653-4).

\section{Appendix A.}

Here, we derive general formulas for Gaussian integrals over a mixed symmetry tensor $\omega_{\mu \nu \rho}$. Let us start from the general expression

$$
\mathcal{L}_{\omega F}=k \omega_{\alpha \beta \gamma}^{2}+r \omega_{\alpha \beta \gamma} \omega^{\gamma \beta \alpha}+s \omega_{\mu} \omega^{\mu}+2 \omega_{\alpha \beta \gamma} F^{\alpha \beta \gamma}
$$

where $F_{\mu \nu \rho}$ is arbitrary except for $F_{\mu \nu \rho}=-F_{\mu \rho \nu}$. In order to Gaussian integrate over $\omega_{\mu \nu \rho}$ we introduce a tensor $T_{\mu \nu \rho}=-T_{\mu \rho \nu}$ which must be determined as a function of $F_{\mu \nu \rho}$ such that the Lagrangian $\mathcal{L}_{\omega F}$ can be written as 


$$
\begin{aligned}
\mathcal{L}_{\omega F}= & k\left(\omega_{\alpha \beta \gamma}+T_{\alpha \beta \gamma}\right)^{2}+r\left(\omega_{\alpha \beta \gamma}+T_{\alpha \beta \gamma}\right)\left(\omega^{\gamma \beta \alpha}+T^{\gamma \beta \alpha}\right) \\
& +s\left(\omega_{\mu}+T_{\mu}\right)^{2}-T_{\alpha \beta \gamma}\left[k T^{\alpha \beta \gamma}+\frac{r}{2}\left(T^{\gamma \beta \alpha}-T^{\beta \gamma \alpha}\right)+\frac{s}{2}\left(\eta^{\alpha \beta} T^{\gamma}-\eta^{\alpha \gamma} T^{\beta}\right)\right] .
\end{aligned}
$$

Comparing the linear terms on $\omega_{\alpha \beta \gamma}$ in (71) and (72), we must have

$$
F^{\alpha \beta \gamma}=k T^{\alpha \beta \gamma}+\frac{r}{2}\left(T^{\gamma \beta \alpha}-T^{\beta \gamma \alpha}\right)+\frac{s}{2}\left(\eta^{\alpha \beta} T^{\gamma}-\eta^{\alpha \gamma} T^{\beta}\right) .
$$

After the shift $\omega_{\alpha \beta \gamma} \rightarrow \tilde{\omega}_{\alpha \beta \gamma}-T_{\alpha \beta \gamma}$ in (72), the field $\tilde{\omega}_{\alpha \beta \gamma}$ decouples and the Gaussian integral over $\omega_{\mu \nu \rho}$ and leaves us with the last term of (72). Using (73) we have

$$
\mathcal{L}_{F F}=-F^{\mu \nu \rho} T_{\mu \nu \rho} \text {. }
$$

All we need to know is how $T_{\mu \nu \rho}$ depends on $F_{\mu \nu \rho}$. From the trace of (73) we have

$$
F^{\gamma}=\frac{1}{2}[2 k+r+(D-1) s] T^{\gamma} .
$$

Since, in general, $\eta^{\mu \nu} F_{\mu \nu \rho} \equiv F_{\rho} \neq 0$, we assume henceforth

$$
2 k+r+(D-1) s \neq 0 .
$$

From (75) back in (73) we have

$$
G^{\alpha \beta \gamma} \equiv F^{\alpha \beta \gamma}+\frac{s}{2 k+r+(D-1) s}\left(\eta^{\alpha \gamma} F^{\beta}-\eta^{\alpha \beta} F^{\gamma}\right)=k T^{\alpha \beta \gamma}+\frac{r}{2}\left(T^{\gamma \beta \alpha}-T^{\beta \gamma \alpha}\right) .
$$

Taking into account all cyclic combinations of (77) we build up a linear system of three equations from which a matrix inversion allows us to obtain $\left(T^{\alpha \beta \gamma}, T^{\gamma \beta \alpha}, T^{\beta \gamma \alpha}\right)$ in terms of $\left(G^{\alpha \beta \gamma}, G^{\gamma \beta \alpha}, G^{\beta \gamma \alpha}\right)$. In particular,

$$
T^{\alpha \beta \gamma}=\frac{1}{(k-r)(2 k+r)}\left[(2 k-r) G^{\alpha \beta \gamma}-r G^{\gamma \beta \alpha}+r G^{\beta \gamma \alpha}\right],
$$

we have assumed:

$$
(k-r)(2 k+r) \neq 0 .
$$

From (78) back in $\mathcal{L}_{F F}$ we finally obtain the result of the Gaussian integral over $\omega_{\mu \nu \rho}$ in (71), i.e.,

$$
\mathcal{L}_{F F}=\frac{1}{(r-k)(r+2 k)}\left\{(2 k-r) F_{\alpha \beta \gamma}^{2}-2 r F_{\alpha \beta \gamma} F^{\gamma \beta \alpha}+\frac{4 s(r-k)}{[2 k+r+s(D-1)]} F_{\gamma}^{2}\right\} .
$$

If we take $F_{\mu \nu \rho}$ as general as the one given in (9) we have, after several rearrangements, a Lagrangian density of the type given in (4) where:

$$
\begin{gathered}
a_{1} L=2\left(2 a b-c^{2}\right)(2 k-r)-2 r\left[a^{2}+b^{2}+2 c(a-b)\right]+R(a+b)^{2}+V t^{2}+W t \\
a_{2} L=4(2 k-r)(a c-a b-b c)+4 r\left(a^{2}+b^{2}+c^{2}-a b-b c+a c\right) \\
-2 R(a-c)(a+b)+2 V u t+u W+t Z \\
a_{3} L=-2(2 k-r)\left(b^{2}+2 a c\right)-2 r\left[a^{2}+c^{2}-2 b(a+c)\right] \\
+R(c-a)^{2}+V u^{2}+u Z
\end{gathered}
$$




$$
\begin{aligned}
b_{1} L & =d(Z+W)-R(b+c)^{2}-V d^{2}, \\
b_{2} L & =2 d(t+u) V+(d-t-u)(Z+W)-2 R(b+c)^{2}, \\
p_{+} & \equiv \frac{p_{1}+p_{2}}{2}=\frac{4(b+c)^{2}}{r+2 k} ; \quad p_{-} \equiv \frac{p_{1}-p_{2}}{2} \\
& =\frac{4}{3}\left[\frac{(b-c+2 a)^{2}}{r+2 k}-\frac{(b-c-a)^{2}}{r-k}\right],
\end{aligned}
$$

and we have defined

$$
\begin{aligned}
& L=(r-k)(r+2 k), \\
& R=\frac{4 s(r-k)}{2 k+r+s(D-1)}, \\
& V=(D-1)[R(D-1)+4(k-r)], \\
& W=8(a+b)(k-r)+2(D-1)(a+b) R, \\
& Z=2(c-a)[4(k-r)+(D-1) R] .
\end{aligned}
$$

\section{Appendix B.}

Let us define the master action:

$\mathcal{L}_{M}[\bar{\Omega}, \bar{\omega}]=\frac{1}{2} \partial^{\gamma} \bar{\Omega}_{\mu \nu \gamma} \partial_{\gamma} \bar{\Omega}^{\nu \mu \gamma}+\frac{m^{2}}{2} \bar{\Omega}_{\alpha \beta \gamma} \bar{\Omega}^{\gamma \beta \alpha}-\frac{1}{2} \partial^{\gamma}\left(\bar{\Omega}_{\mu \nu \gamma}-\bar{\omega}_{\mu \nu \gamma}\right) \partial_{\gamma}\left(\bar{\Omega}^{\nu \mu \gamma}-\bar{\omega}^{\nu \mu \gamma}\right)$,

After the shift $\bar{\omega}_{\mu \nu \rho} \rightarrow \bar{\omega}_{\mu \nu \rho}+\bar{\Omega}_{\mu \nu \rho}$ it is clear that $\mathcal{L}_{M}[\bar{\Omega}, \bar{\omega}]$ has the same particle content of (28), since $\partial^{\gamma} \bar{\omega}_{\mu \nu \gamma} \partial_{\gamma} \bar{\omega}^{\nu \mu \gamma}$ has no particle content as we have proved in subsection 4.3. On the other hand, we can write

$$
\begin{aligned}
& \mathcal{L}_{M}[\bar{\Omega}, \bar{\omega}]=\frac{m^{2}}{2} \bar{\Omega}_{\alpha \beta \gamma} \bar{\Omega}^{\gamma \beta \alpha}-\bar{\Omega}_{\alpha \beta \gamma} \partial^{\gamma} \partial_{\rho} \bar{\omega}^{\beta \alpha \rho}-\frac{1}{2} \partial^{\gamma} \bar{\omega}_{\mu \nu \gamma} \partial_{\gamma} \bar{\omega}^{\nu \mu \gamma}, \\
& =\frac{m^{2}}{2} \bar{\Omega}_{\alpha \beta \gamma} \bar{\Omega}^{\gamma \beta \alpha}+2 \bar{\Omega}_{\alpha \beta \gamma} \bar{F}^{\alpha \beta \gamma}-\frac{m^{2}}{2} \bar{e}_{\mu \nu} \bar{e}^{\nu \mu}
\end{aligned}
$$

where

$$
\begin{aligned}
& \bar{e}^{\mu \nu}=\frac{1}{m} \partial_{\rho} \bar{\omega}^{\mu \nu \rho}, \\
& \bar{F}^{\alpha \beta \gamma}=\frac{m}{4}\left(\partial^{\beta} \bar{e}^{\gamma \alpha}-\partial^{\gamma} \overline{\boldsymbol{e}}^{\beta \alpha}\right) .
\end{aligned}
$$

In order to integrate over $\bar{\Omega}_{\alpha \beta \gamma}$ we use the general result (80) with $(k, r, s)=\left(0, m^{2} / 2,0\right)$ and obtain the fourth-order model

$$
\mathcal{L}^{(4)}=\left(\partial^{\mu} \bar{e}_{(\mu \nu)}\right)^{2}+\frac{1}{2} \bar{e}_{(\mu \nu)} \square \bar{e}^{(\mu \nu)}-\frac{m^{2}}{2} \bar{e}_{\mu \nu} \bar{e}^{\nu \mu} .
$$


In $D=2+1$ we can always write without loss of generality $\bar{\omega}_{\mu \nu \rho}=\epsilon_{\nu \rho \beta} h_{\mu}^{\beta}$, where $h_{\beta \mu}=h_{\mu \beta}$ follows from the traceless condition $\eta^{\mu \nu} \bar{\omega}_{\mu \nu \rho}=0$. In this case $\mathcal{L}^{(4)}$ becomes the linearized version of the NMG of [8] with $g_{\mu \nu}=\eta_{\mu \nu}+h_{\mu \nu}$. In $D=3+1$ the theory $\mathcal{L}^{(4)}$ is the linearized 4D NMG suggested in [12]. While in arbitrary $D$-dimensions we have the linearized $D$-dimensional NMG of $[14,18]$. As explained in [14], $\mathcal{L}^{(4)}$ corresponds to the usual massive FP model with the replacement $e_{\mu \nu} \rightarrow \partial^{\rho} \bar{\omega}_{\mu \nu \rho} / m$ directly at action level.

\section{Appendix C.}

Let us require that $\mathcal{L}_{\omega e}(m=0)$, see $(7)$, be invariant, up to total derivatives, under the vector transformations:

$$
\begin{gathered}
\delta_{\xi} e_{\alpha \beta}=\partial_{\beta} \xi_{\alpha} \\
\delta_{\xi} \omega_{\alpha \beta \gamma}=A \partial_{\alpha}\left(\partial_{\beta} \xi_{\gamma}-\partial_{\gamma} \xi_{\beta}\right)+B\left(\eta_{\alpha \beta} \partial_{\gamma}-\eta_{\alpha \gamma} \partial_{\beta}\right) \partial \cdot \xi+C\left(\eta_{\alpha \beta} \square \xi_{\gamma}-\eta_{\alpha \gamma} \square \xi_{\beta}\right) .
\end{gathered}
$$

In the transformations (99) the constants $A, B, C$ are so far arbitrary. Requiring $\delta_{\xi} \mathcal{L}_{\omega e}=0$ at $m=0$ gives rise to several equations. Two of them can be written as

$$
A(a+b)=0 ; \quad A(a-c)=0 .
$$

From (5) we have $p_{+}=\left(p_{1}+p_{2}\right) / 2=1 / 2$, back in (86) we see that $b+c \neq 0$, therefore from the difference of the equations (100) it follows that $A=0$. Now, the cancelation of all terms of the type $\omega \partial^{2} \xi$ in $\delta_{\xi} \mathcal{L}_{\omega e}$ at $m=0$ can be used to fix $a, B$ and $C$ as follows:

$a=c ; \quad B=-\frac{2(d+t)}{2 k+r+s(D-1)} ; \quad C=-\frac{2 u}{2 k+r+s(D-1)}$.

The cancelation of the remaining terms and the above formulas lead to our final conditions:

$$
\begin{aligned}
(d+t)(d+t+u)=0 ; & u[b+c+t(D-1)]=0, \\
(d+t)[b+c+t(D-1)]=0 ; & u[b-u(D-1)]=0, \\
(d+t)[b-u(D-1)]=0 &
\end{aligned}
$$

We have three cases:

1. $d+t=0 ; \quad \mathrm{u}=0$

2. $d+t=0 ; \quad b=(D-1) u ; \quad b+c=(D-1) d$.

3. $d+t=-u ; \quad b=(D-1) u ; \quad c=(D-1) d$.

In what follows, we find $r$ and $k$ from the two formulas (86) with $\left(p_{+}, p_{-}\right)=(1 / 2,0)$, see (5), and plug them back in (81)-(85). If we use the case I conditions $d+t=0=u$, we exactly reproduce the family $\mathcal{L}_{a_{1}}$ in the last row of table 1 after formula (5). It is necessary to assume that $b+c \neq(D-1) d$.

On the other hand, in case II it turns out that $a_{1}=-\frac{D-3}{4(D-1)}$. From the second row of table 1 we should have $S=a_{1}+a_{2}+a_{3}=D /[2(D-1)]$, however this is only true if $u=0$.

At last, in case III we obtain $S=a_{1}+a_{2}+a_{3}=D /[2(D-1)]$ but $\left(a_{2}, a_{3}\right)$ differ from $(1 / 2,1 / 4)$, see $(5)$, by terms proportional to $u$. So once again we must have $u=0$. This completes the proof of (12). 


\section{References}

[1] Hooft G 't and Veltman M J G 1974 Annales Poincare Phys. Theor. A 20 69-94

[2] Goroff M H and Sagnotti A 1986 Nucl. Phys. B 266709

[3] Stelle K S 1977 Phys. Rev. D $16953-69$

[4] Deser S, Jackiw R and Templeton S 1982 Annals Phys. 140 372-411

Erratum-ibid Deser S, Jackiw R and Templeton S 1988 Annals Phys. 185406

Deser S, Jackiw R and Templeton S 2000 Annals Phys. 281 409-49

[5] Hinterbichler K 2012 Rev. Mod. Phys. 84 671-710

[6] de Rham C 2014 Living Rev. Relativity 177

[7] Sbisa F 2014 Modified theories of gravity arXiv:1406.3384

[8] Bergshoeff E, Hohm O and Townsend P K 2009 Phys. Rev. Lett. 102201301

[9] Blagojevic M and Cvetkovic B 2011 J. High Energy Phys. JHEP01(2001)082

[10] de Rham C, Gabadadze G, Pirtskhalava D, Tolley A J and Yavin I 2011 J. High Energy Phys. JHEP06(2011)028

[11] Muneyuki K and Ohta N 2012 Phys. Rev. D 85101501

[12] Bergshoeff E, Fernandez-Melgarejo J J, Rosseel J and Townsend P K 2012 J. High Energy Phys. JHEP04(2012)070

[13] Deser S 2009 Phys. Rev. Lett. 103101302

[14] Dalmazi D and Santos R C 2013 Phys. Rev. D 87085021

[15] Fierz M and Pauli W 1939 Proc. Roy. Soc. Lond. A 173211

[16] Curtright T L and Freund P G O 1980 Nucl. Phys. B 172413

[17] Gonzalez B, Khoudeir A, Montemayor R and Urrutia L F 2008 J. High Energy Phys. JHEP09 (2008)058

[18] Joung E and Mkrtchyan K 2013 J. High Energy Phys. JHEP02(2013)134

[19] Morand K and Solodukhin S N 2012 Phys. Lett. B 715 260-6

[20] Dalmazi D 2013 Phys. Rev. D 87125027

[21] Dalmazi D 2013 Phys. Rev. D 88045003

[22] Dalmazi D, dos Santos A L R and Mendonça E L 2015 Annals Phys. 354 385-93

[23] Zinoviev Y M 2005 J. High Energy Phys. JHEP10(2005)075

[24] Deser S and Jackiw R 1984 Phys. Lett. B 139371

[25] Dalmazi D and Mendonça E L 2009 J. High Energy Phys. JHEP09(2009)011

[26] Andringa R, Bergshoeff E A, de Roo M, Hohm O, Sezgin E and Townsend P K 2010 Class. Quant. Grav. 27025010 